\title{
Primera serie dendroarqueológica en los Andes Centrales: resultados preliminares de Chankillo,
} Casma

Premières séries dendroarchéologiques dans les Andes Centrales : résultats préliminaires de Chankillo, Casma

The First dendroarchaeological series in the Central Andes: Preliminary results from Chankillo, Casma

Iván Ghezzi y Rodolfo Rodríguez

\section{OpenEdition}

Journals

Edición electrónica

URL: http://journals.openedition.org/bifea/7465

DOI: 10.4000/bifea.7465

ISSN: 2076-5827

Editor

Institut Français d'Études Andines

Edición impresa

Fecha de publicación: 1 abril 2015

Paginación: 1-21

ISSN: 0303-7495

Referencia electrónica

Iván Ghezzi y Rodolfo Rodríguez, « Primera serie dendroarqueológica en los Andes Centrales: resultados preliminares de Chankillo, Casma », Bulletin de I'Institut français d'études andines [En línea], 44 (1) | 2015, Publicado el 08 mayo 2015, consultado el 05 noviembre 2020. URL : http:// journals.openedition.org/bifea/7465; DOI : https://doi.org/10.4000/bifea.7465

\section{(c)}

Les contenus du Bulletin de l'Institut français d'études andines sont mis à disposition selon les termes de la licence Creative Commons Attribution - Pas d'Utilisation Commerciale - Pas de Modification 4.0 International. 


\title{
Primera serie dendroarqueológica en los Andes Centrales: resultados preliminares de Chankillo, Casma
}

\author{
Iván Ghezzi* \\ Rodolfo Rodríguez ${ }^{*}$
}

\section{Resumen}

La dendrocronología empieza a dar sus frutos en el Perú: las investigaciones recientes muestran que el crecimiento de los anillos del algarrobo (Prosopis sp.) cumple con los requisitos básicos de esta disciplina. El objetivo del trabajo es elaborar una serie dendroarqueológica a partir del estudio de los dinteles de algarrobo prehispánicos del sitio Chankillo, Ancash. En primer lugar, el estudio de árboles modernos de Casma reportado aquí demuestra una alta correlación entre el ancho de los anillos de crecimiento del algarrobo y registros climáticos de los últimos 100 años. El principio de uniformitarianismo indica que en tiempos prehispánicos ocurriría lo mismo. En segundo lugar, en Chankillo, sitio con numerosos dinteles de algarrobo in situ y bien conservados, el muestreo y medición dendrocronológica de la madera de los dinteles ha permitido crear la primera serie dendroarqueológica de los Andes Centrales. La secuencia es flotante, por lo que aún no proporciona fechas, pero sí permite una aproximación a las variaciones climáticas anuales de la época, en especial el fenómeno El Niño. Además, ilustra la cronología de la construcción del sitio. La perspectiva a futuro es elaborar una secuencia maestra para toda la costa peruana, que abarque desde las primeras construcciones monumentales hasta el presente, y que tendría aplicaciones importantes en Arqueología, Climatología, y otras ciencias.

Palabras clave: dendrocronología, dendroarqueología, algarrobo, Prosopis sp., Chankillo, El Niño, ENSO

* Instituto de Investigaciones Arqueológicas, La Conquista 296, Of. D202, Surco, Lima 33. E-mail: ighezzi@idarq.org

** Laboratorio de Dendrocronología, Universidad de Piura, Av. Ramón Mugica 131, Piura. E-mail: rodolfo.rodriguez@udep.pe 


\title{
Premières séries dendroarchéologiques dans les Andes Centrales : résultats préliminaires de Chankillo, Casma
}

\section{Résumé}

La dendroarchéologie commence à porter ses fruits au Pérou : les recherches récentes démontrent que la croissance des anneaux de l'algarrobo (Prosopis sp.) satisfait les exigences de base de cette discipline. Cette étude vise à élaborer une série dendroarchéologique à partir de l'étude des linteaux d'algarrobo préhispaniques du site de Chankillo, Ancash. Tout d'abord, I'étude des arbres modernes de Casma ici rapportée met en évidence une forte corrélation entre la largeur des anneaux de croissance de l' algarrobo et les registres climatiques des cent dernières années. Selon le principe de I'uniformitarisme il serait arrivé la même chose dans les temps préhispaniques. Ensuite, à Chankillo, un site présentant de nombreux linteaux en algarrobo in situ bien conservés, l'échantillonnage et la mesure dendrochronologique du bois des linteaux a permis de créer la première série dendroarchéologique des Andes centrales. II s'agit d'une séquence flottante qui ne fournit pas encore des dates, mais qui permet une approximation des variations climatologiques annuelles de l'époque, surtout du phénomène El Niño. En outre, elle sert à éclairer la chronologie de la construction du site. À terme il s'agit d'élaborer une séquence maîtresse pour toute la côte péruvienne couvrant depuis les toutes premières constructions monumentales jusqu'àux actuelles, et qui pourrait avoir d'importantes applications en archéologie, en climatologie et dans d'autres sciences.

Mots-clés : dendrochronologie, dendroarchéologie, algarrobo, Prosopis sp., Chankillo, El Niño, ENSO

\section{The First dendroarchaeological series in the Central Andes: Preliminary results from Chankillo, Casma}

\begin{abstract}
Dendrochronology has begun to yield results in Peru. Recent research shows that the growth of rings from the algarrobo (Prosopis sp.) tree meets basic requirements for its use in the discipline. The goal of this research is to create a dendroarchaeological series of the algarrobo lintels from the Chankillo archaeological site in coastal Ancash. A study of modern algarrobos in Casma reported here shows a high correlation between ring-widths and climatic records from the past century. The principle of uniformitarianism dictates the same was true in pre-Hispanic times. At Chankillo, an archaeological site with numerous in situ and well-preserved lintels made from algarrobo wood, our sampling and dendrochronological measurements have yielded the first dendroarchaeological series in the Central Andes. It is only a floating sequence, without the ability to give dates yet, but it allows an approximation of the annual climatic variations of the time, in particular the El Niño phenomenon, and it also illustrates the chronology of construction of the site. The outlook for the future is to elaborate a master dendrochronological sequence for coastal Peru, from the earliest monumental constructions to the present, which would have important applications in archaeology, climatology, and other disciplines.
\end{abstract}

Keywords: Dendrochronology, Dendroarchaeology, Algarrobo, Prosopis sp., Chankillo, El Niño, ENSO 


\section{INTRODUCCIÓN}

La dendrocronología aprovecha parámetros mensurables en la estructura del anillado de los árboles, como el ancho de los anillos, la densidad de la madera, y la concentración de algún compuesto químico o isótopo, para elaborar secuencias cronológicas absolutas que permiten fechar el año de formación de cada anillo. Sus resultados superan a otros métodos de fechado, como el radiocarbono, por su precisión anual y sin error estadístico. Esta precisión calendárica permite elaborar cronologías detalladas, y el estudio a largo plazo de procesos ambientales y culturales relativos a la adaptación humana a una región, como las variaciones climáticas, los cambios ecológicos, y el impacto humano en el ambiente (Douglass, 1919; Stokes \& Smiley, 1968; Bannister, 1969; Ferguson, 1970; Fritts, 1976; Schweingruber, 1988; Baillie, 1995; Dean, 1997; McCarroll \& Loader, 2004; Rodríguez \& Fernández, 2009; Speer, 2010). En la Arqueología se usa principalmente para determinar con precisión la fecha en la que un árbol fue derribado y su madera usada en la construcción de edificios o en la fabricación de artefactos (Douglass, 1929; Dean, 1978; Baillie, 1982; Billamboz, 1992; Towner, 2001; Ghezzi et al., 2002; Sass-Klaassen, 2002; Towner, 2002; Billamboz, 2003; Čufar, 2007; Dean et al., 2009; Grissino-Mayer, 2009; Morales, et al., 2013). También se usa secuencias dendrocronológicas para construir las curvas que permiten calibrar los fechados radiocarbónicos (Salcedo, 2011; Hogg et al., 2013; Reimer et al., 2013).

En este artículo se presenta la primera serie dendroarqueológica elaborada en el Perú a partir de madera de algarrobo de Chankillo, un sitio arqueológico en Casma, Ancash. Para entender la importancia de este resultado es útil conocer su contexto, aunque no se encuentra entre los objetivos del artículo hacer un recuento histórico de la dendrocronología, que puede consultarse en otras fuentes (Speer, 2010). La dendrocronología se desarrolló primero en zonas del mundo en que la marcada estacionalidad provoca que los árboles frecuentemente tengan anillos anuales de crecimiento visibles, cuyo grosor responde a la variación climática (Douglass, 1919; 1937; Fritts, 1976). Por el contrario, para las zonas tropicales y subtropicales se sostuvo durante mucho tiempo que la dendrocronología estaba restringida, por el bajo número de especies que producen anillos anuales de crecimiento, por su corta longevidad, y por la pobre preservación de la madera. Este prejuicio, defendido hasta hace poco (Towner, 2001; Speer, 2010), produjo un retraso de décadas en la aplicación de la dendrocronología en países como el Perú, donde podría dar aportes importantes.

Felizmente, las investigaciones recientes en regiones de Sudamérica antes ignoradas por la dendrocronología han empezado a corregir el error (Boninsegna et al., 2009). Hay numerosos reportes de anillos de crecimiento visibles, anuales, e influenciados por el clima, en árboles del género Prosopis de Argentina, Bolivia, Chile y Perú (Villalba et al., 2000; Morales et al., 2001; Ghezzi et al., 2002; López et al., 2005; Rodríguez et al., 2005; Christie et al., 2009; Rivera et al., 2010; Brienen et al., 2012; Morales et al., 2012). Por lo tanto, hay un gran potencial para el desarrollo de secuencias dendrocronológicas en zonas donde crecen 
las especies del género. Además, se cuenta con fechas dendroarqueológicas de contextos prehistóricos (chullpas) en el altiplano boliviano (Morales et al., 2013).

Gracias a una nueva postura en la dendrocronología, más favorable al estudio de especies tropicales y subtropicales, específicamente el algarrobo (Prosopis sp.), Chankillo presenta gran potencial de estudio, por la abundante madera usada en su construcción. Por lo tanto, el objetivo principal del proyecto cuyos primeros resultados se reporta en este artículo es aprovechar la presencia en Chankillo de numerosos dinteles de madera de algarrobo, in situ y en buen estado de conservación, para elaborar la primera secuencia dendroarqueológica de los Andes Centrales.

\section{EL SITIO ARQUEOLÓGICO CHANKILLO}

Chankillo es un gran complejo ceremonial con atributos de observación astronómica (Ghezzi \& Ruggles, 2007), rituales y de defensa, construido en el desierto costero adyacente a los valles irrigados del ramal sur de la cuenca del río Casma/Sechín, frente a las escarpadas laderas occidentales andinas, en el distrito y provincia de Casma, región Ancash (fig. 1).

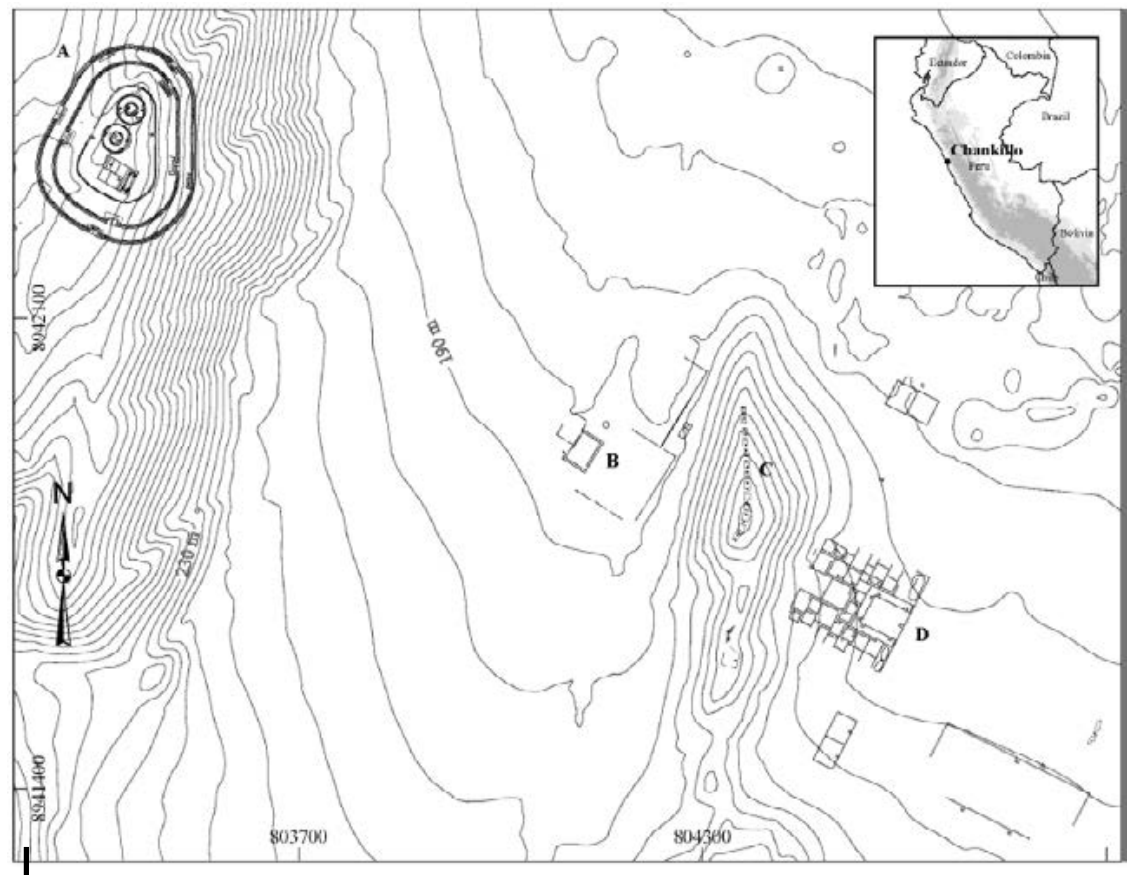

Figura 1 - Mapa arqueológico de los principales complejos de edificios en Chankillo, entre ellos: (A) el Templo Fortificado, (B) el Observatorio, (C) las Trece Torres y (D) el Complejo Administrativo.

Coordenadas UTM, Zona 17S, WGS 1984

(C) Iván Ghezzi 
El sitio se extiende sobre $4 \mathrm{~km}^{2}$ de afloramientos de roca, pampas desérticas y dunas de arena, entre los 80 y 330 m de altitud. Es parte de un paisaje de colinas, valles y llanuras, que se cuenta entre los más secos del mundo, y se mantiene prácticamente sin mayor cambio geológico desde el Pleistoceno (Ghezzi \& Guadalupe Gómez, 2013). Como en toda la costa peruana, los valles de Casma y Sechín han sido durante largo tiempo un «oasis» en el desierto, que posibilita el asentamiento de poblaciones en un territorio inhóspito.

En Chankillo, a 14 km del litoral, el viento avanza en dirección noreste alcanzando velocidades de hasta $25 \mathrm{kph}$. Se presenta una temperatura media de $240 \mathrm{C}$ en verano y $19^{\circ} \mathrm{C}$ en invierno. En la franja de desierto costero contigua a los campos de cultivo, en el lado sur del valle de Casma, entre las localidades de La Cantina y Nivín, se ubican poblaciones dispersas de algarrobo. Aunque en el valle existen muchas otras especies arbóreas, el bosque seco está formado exclusivamente por algarrobos bien adaptados a este ecosistema árido.

El algarrobo es el género dominante de los bosques secos de la costa del Perú. Es una especie arbórea perteneciente a la familia de las fabáceas, la más numerosa e importante del mundo vegetal (Pasiecznik et al., 2001). Su alta resistencia a la salinidad, desde la fase juvenil, explica su adaptación a zonas áridas y semiáridas del continente (Palacios et al., 2012). Durante la época de ocupación prehispánica de Chankillo probablemente las poblaciones dispersas de algarrobo formaban un bosque denso, y su madera y fruto se habrían explotado en gran medida, por ejemplo para las construcciones en Chankillo (Salazar, 2013).

Treinta y dos fechados ${ }^{14} \mathrm{C}$ procedentes de excavaciones arqueológicas en distintos sectores de Chankillo (fig. 2) fueron tomados principalmente de restos de plantas de vida corta, minimizando así el problema de la «madera vieja» (Dean, 1978; Schiffer, 1986), una distorsión especialmente problemática en la costa del Perú. Asimismo, cinco de las muestras (AA57020, AA57022-25) fueron obtenidas usando principios dendrocronológicos del anillo externo, o de corte, de los dinteles de madera de algarrobo conservados in situ en Chankillo. En principio, la fecha absoluta de estas muestras debería aproximarse mucho a la fecha de tala del árbol, proporcionando así una excelente datación de la construcción del sitio. Sin embargo, la calibración de los fechados ${ }^{14} \mathrm{C}$ para esta época crea distribuciones de probabilidad con un rango bastante amplio (Salcedo, 2011), de manera que la construcción, ocupación y abandono de Chankillo se fechan aproximadamente entre los años 400 y 100 CalBC, correspondientes a la fase tardía del periodo Horizonte Temprano (Burger, 1995).

Chankillo comprende varias construcciones monumentales de piedra y mortero de barro. Una de las más sobresalientes es una estructura de forma ovoide ubicada en la cima de un cerro, a $180 \mathrm{~m}$ sobre el fondo del valle, que se conoce localmente como La Fortaleza (fig. 3). Es una construcción masiva, de $300 \mathrm{~m}$ de longitud que destaca no solo por su ubicación defensiva, sino también por un nivel de fortificación apreciable que protege tres edificios centrales con murallas concéntricas de hasta $8 \mathrm{~m}$ de alto, grandes paredes, portadas monumentales, accesos restringidos, parapetos, atalayas, etc. La función de este edificio ha sido 


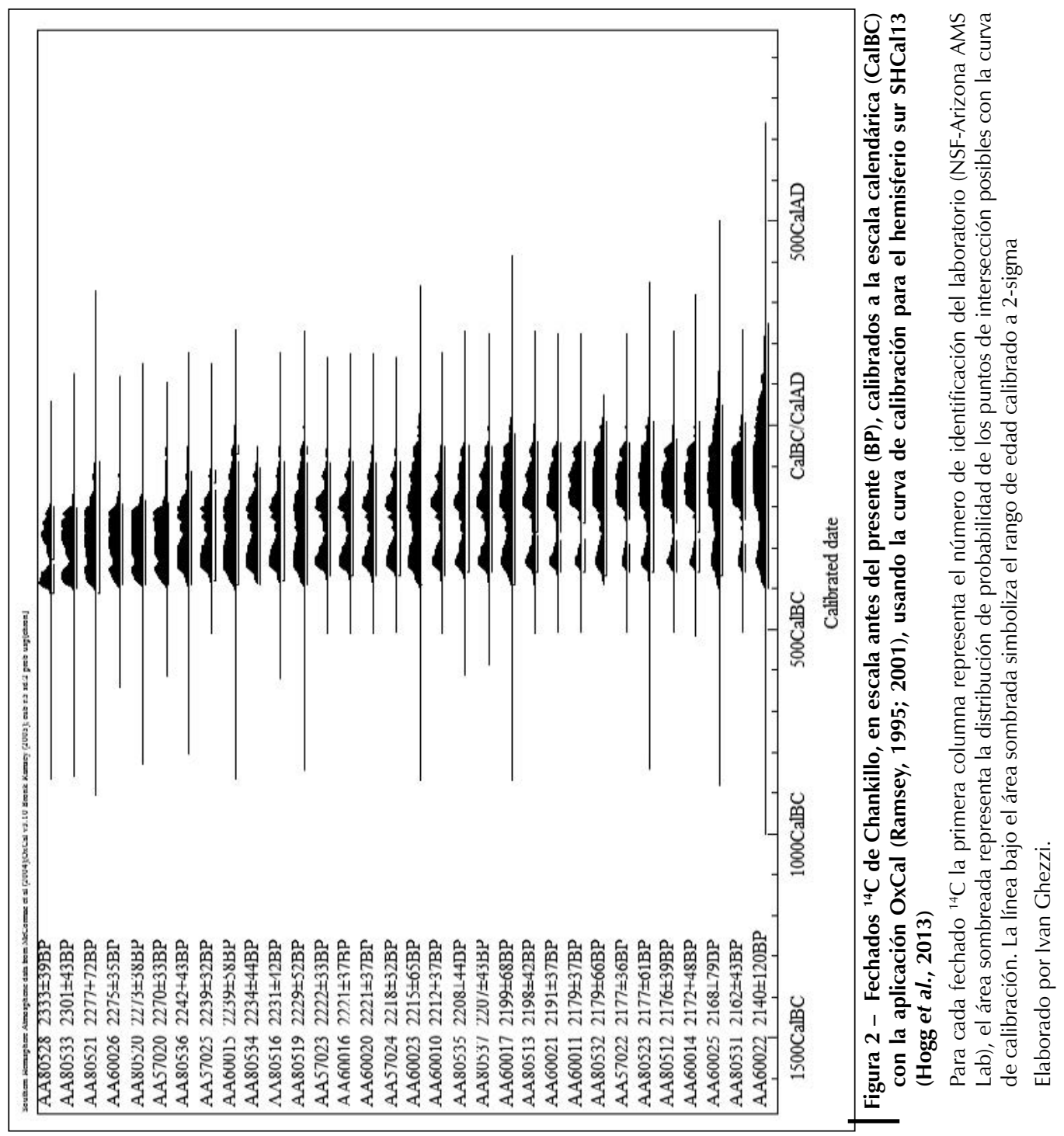




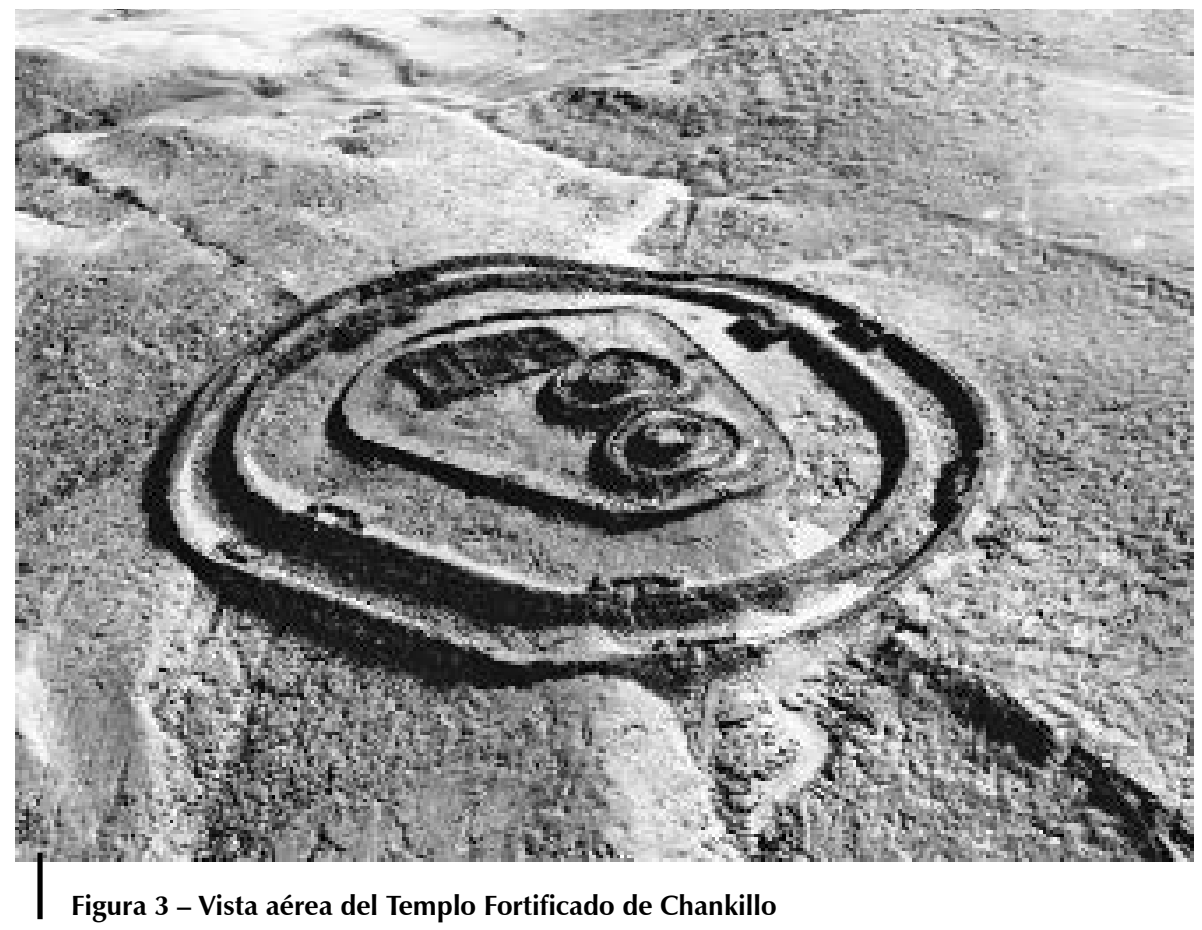

(C) Servicio Aerofotográfico Nacional

objeto de un considerable debate, pues ha sido descrito como una fortaleza, un reducto, o un sitio ceremonial para combates rituales. Las más recientes investigaciones apoyan una interpretación alternativa de este edificio como un «templo fortificado», construido en un tiempo en que la guerra tenía motivaciones ideológicas, y los blancos de los ataques y destrucción eran los símbolos y los lugares de poder del adversario (Ghezzi, 2006; 2007).

Las portadas monumentales de acceso a través de las murallas y otros ingresos están techadas con dinteles de madera de algarrobo que soportan rellenos de piedra y grava (fig. 4). Pese al saqueo, aún se conservan decenas de dinteles in situ, formando parte integral de la construcción en pie, y en excelente estado de conservación. Otros dinteles están abandonados entre los muros del edificio, en regular estado de conservación por su prolongada exposición al sol. Además, durante las excavaciones del Proyecto Chankillo (Ghezzi, 2006; 2007) se recuperaron algunos dinteles enterrados bajo los escombros, fuera de su posición original, y en un estado de conservación regular. En total, pudo haber cientos de dinteles de madera de algarrobo tan solo en el Templo Fortificado.

No se sabe a ciencia cierta si los constructores de Chankillo tenían otras opciones además de la madera del algarrobo para estos dinteles. En todo caso, su extraordinaria dureza permitió que soportaran las toneladas de relleno de piedras 


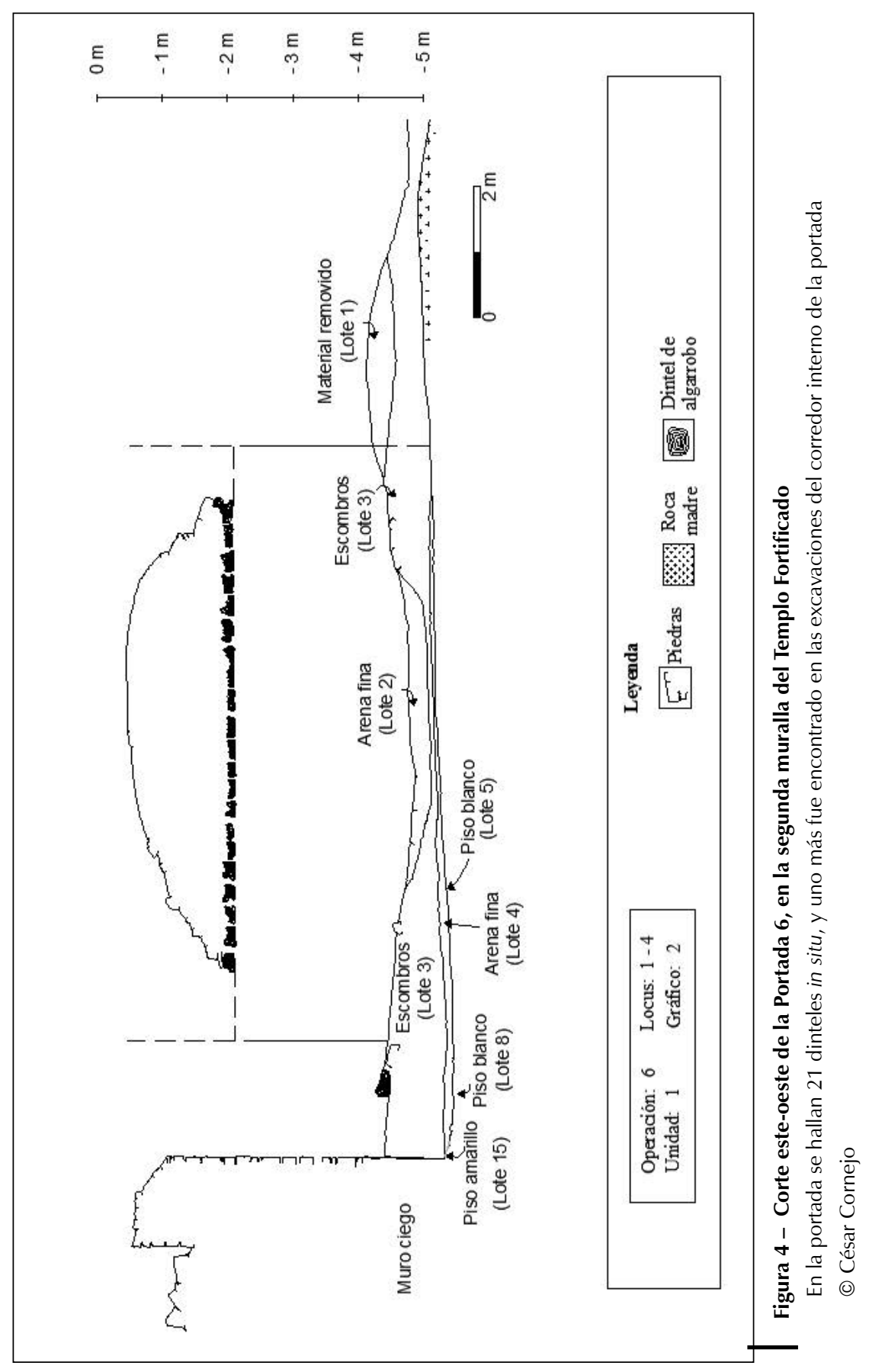


y barro que componen los techos de las portadas monumentales de las murallas. En la muralla intermedia, las excavaciones muestran que las portadas estaban coronadas por atalayas, por lo que la estructura de dinteles soportaba además el peso de los vigías y de las armas y municiones que ellos portaban y almacenaban. Las huellas visibles en los extremos de los dinteles indican que el talado del árbol se realizó combinando golpes de hacha de piedra y el uso del fuego. A continuación, para darle la forma necesaria a los dinteles, se rebajó el tronco de un solo lado mediante golpes de hacha que dejaron una superficie relativamente plana pero cubierta de marcas ondulantes, producto de la percusión (fig. 5A). El resto del tronco se dejó intacto, incluso la corteza, salvo la remoción ocasional de alguna rama. El lado aplanado de los dinteles se apoyaba contra los muros de la portada, dejando hacia arriba el lado sin trabajar, cubierto de corteza, para soportar el relleno de piedras. La mayoría de los dinteles presenta estos restos de corteza, lo que aumenta enormemente su potencial dendrocronológico, pues bajo la corteza se halla el último anillo desarrollado por el árbol antes de ser talado, el cual representa la fecha de corte (fig. 5B).

Las investigaciones previas de Rodríguez y sus colegas sobre algarrobos de la región Piura (Rodríguez et al., 1993; López et al., 2005; Rodríguez et al., 2005), han permitido conocer el potencial de esta y otras especies para estudios

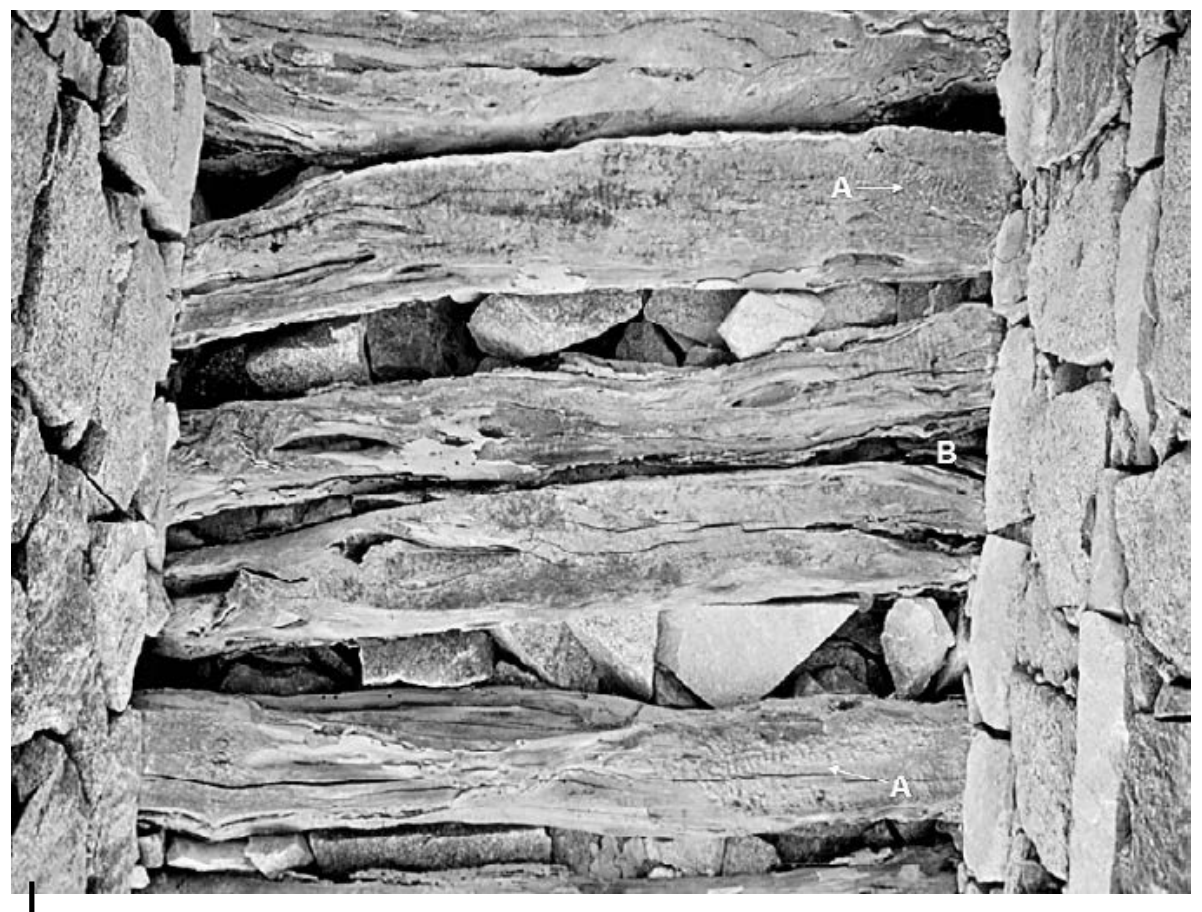

Figura 5 - Foto de un conjunto de dinteles de algarrobo en la Portada 2, primera muralla

Se aprecian las marcas de fabricación (A), y la corteza no removida (B).

(c) Ivan Ghezzi 
dendrocronológicos y, por tanto, para aplicaciones arqueológicas. En el resto de este artículo presentamos los primeros resultados de los estudios dendroarqueológicos realizados en Chankillo.

\section{METODOLOGÍA}

Un paso previo a la elaboración de una secuencia dendroarqueológica a partir de los dinteles de algarrobo de Chankillo consiste en evaluar su aplicabilidad a estos materiales. Primero es necesario demostrar que en esta parte del Perú los árboles de este género en particular reúnen las condiciones básicas para ser usados en investigaciones dendrocronológicas, es decir, que producen anillos visibles y anuales cuyos anchos están correlacionados significativamente con parámetros climáticos de alcance regional (Stokes \& Smiley, 1968). Si esto se comprueba, según el principio de uniformitarianismo (Speer, 2010) podemos asumir que los procesos que gobiernan la respuesta del algarrobo ante las variaciones climáticas no han cambiado significativamente desde la época que se intenta reconstruir —en este caso, la ocupación de Chankillo entre los años 400-100 a. C.- . Por lo tanto, la dendrocronología sería aplicable no solo a los algarrobos modernos en el valle de Casma, sino también a los dinteles prehispánicos de Chankillo.

\section{1. Estudio de algarrobos modernos}

Primero realizamos un estudio de los algarrobos en el bosque seco cercano a Chankillo, para comprobar si, como indica la literatura para otras zonas (Rodríguez et al., 1993; López et al., 2005; Rodríguez et al., 2005), este árbol posee las características necesarias. Para controlar que el clima sea el factor limitante que explica el crecimiento de los anillos en árboles modernos, se seleccionaron individuos creciendo en el desierto, alejados del valle y del sistema de irrigación agrícola, en las condiciones más extremas posibles, que registren la señal climática muy fuertemente (Rodríguez \& Fernández, 2009).

Con una motosierra, o con una sierra manual, se extrajo secciones de 1 pulgada de espesor cada una de 12 algarrobos modernos en el extremo sur del bosque seco cercano a Chankillo. Allí los árboles enfrentan una aridez extrema, y reciben agua solo de las pocas lluvias que ocurren ocasionalmente, y soportando fuertes vientos que acarrean partículas de arena y sales en suspensión (fig. 6).

\section{2. Análisis de material arqueológico}

Para elaborar la secuencia dendroarqueológica se extrajo 130 muestras de los dinteles de algarrobo empleados en las construcciones en el Templo Fortificado (fig. 7). De los dinteles in situ se extrajo tarugos con un taladro especialmente adaptado para este propósito (fig. 8). De los dinteles abandonados entre los muros 


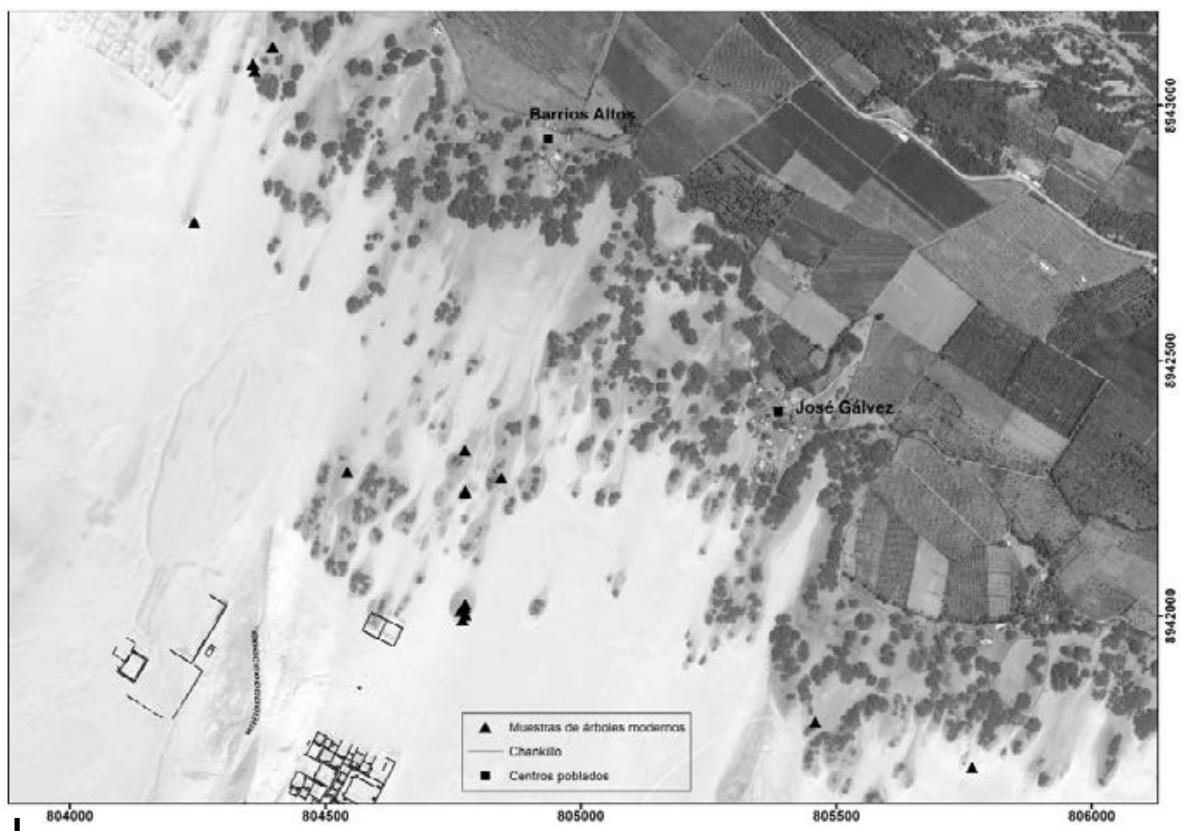

Figura 6 - Mapa de ubicación de la muestras dendrocronológicas tomadas de árboles modernos

(C) Ivan Ghezzi

y los recuperados en mal estado de conservación en las excavaciones se extrajo secciones de alrededor de una pulgada de espesor con una motosierra.

\section{3. Métodos de laboratorio}

En el Laboratorio de Dendrocronología de la Universidad de Piura (UDEP) se prepararon las muestras, siguiendo la metodología estándar aplicada en la dendrocronología. Se observó y analizó la estructura de su anillado y se midió el ancho de los anillos, generando así series dendrocronológicas de valores del ancho de los anillos medidos en las secciones y tarugos, tal como describen Rodríguez \& Fernández (2009).

La preparación de las muestras consistió en secarlas al aire libre y pegarlas con goma soluble en bases de madera, para evitar el deterioro durante su manipulación. El siguiente paso fue pulir la superficie expuesta de las muestras con lijas de diferente granulometría, para maximizar la visibilidad de los anillos.

Para la medición primero se identificó y marcó a lápiz los anillos de crecimiento bajo un microscopio estéreo de alta resolución. Luego, con la ayuda de este microscopio se midió el ancho de los anillos con un sistema de medición Velmex, que es un micrómetro de desplazamiento de una micra de resolución, 


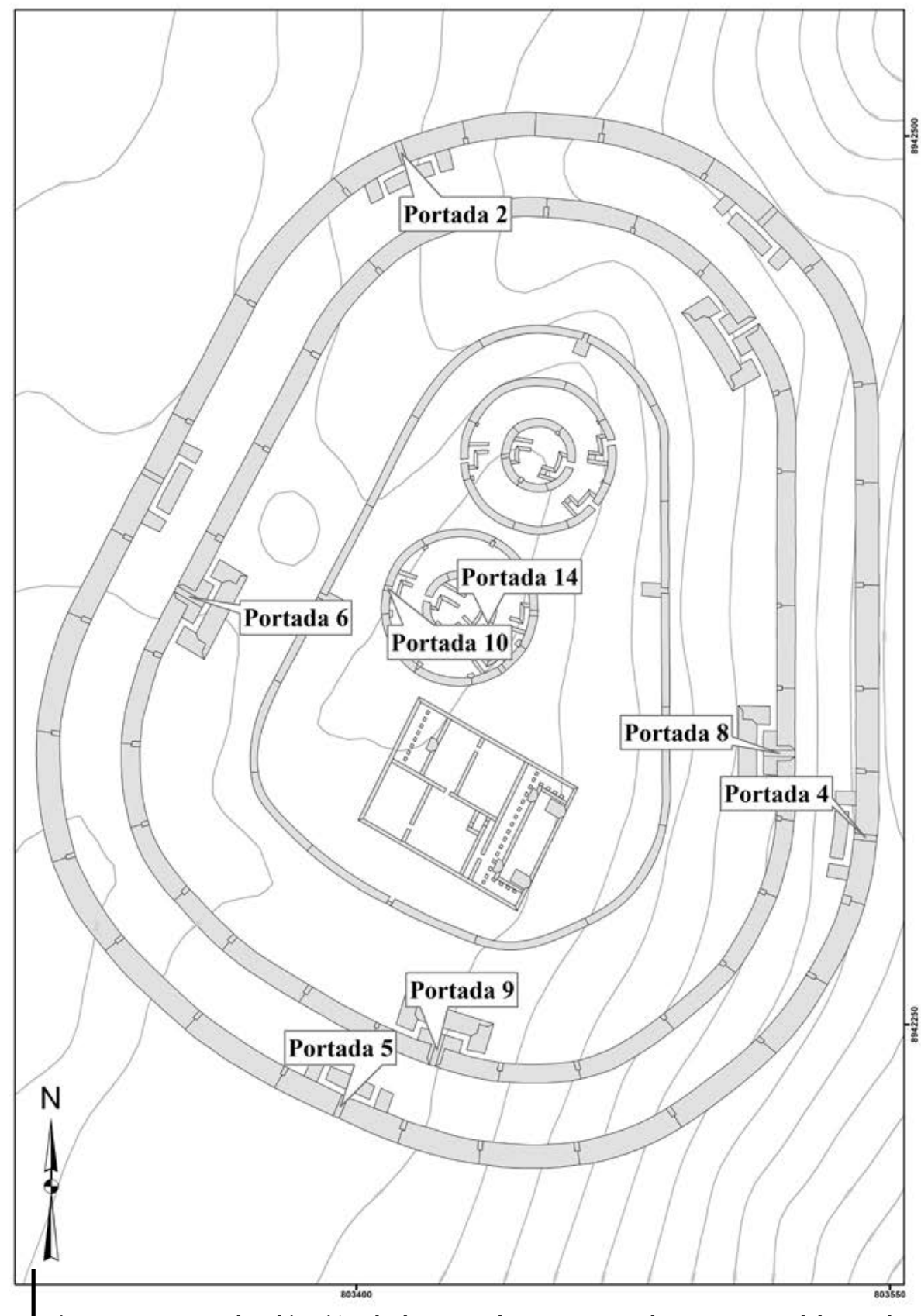

Figura 7 - Mapa de ubicación de las portadas monumentales y accesos del Templo Fortificado en los que se preservan in situ los dinteles de algarrobo muestreados para elaborar la serie dendroarqueológica

(C) Iván Ghezzi 


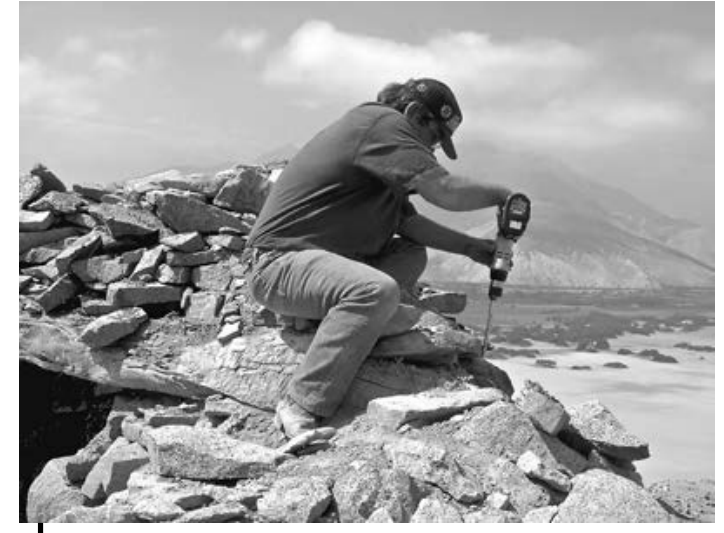

Figura 8 - Extracción de los tarugos con un taladro especialmente adaptado

En la foto Ronald Towner, Laboratory of Tree-Ring Research, Universidad de Arizona, EEUU

(C) Iván Ghezzi provisto de una interface que se conecta a una computadora para la transferencia, almacenamiento, y análisis de los datos.

A continuación se elaboró series individuales para cada muestra con la aplicación ARSTAN (Cook \& Holmes, 1984), que ajusta una función no lineal para estandarizar las series de crecimiento de anillos. El objetivo de la estandarización es minimizar la tendencia atribuida a la edad de los árboles individuales. Se aplicó a continuación la datación cruzada, que establece las coincidencias en el grosor de los anillos entre las muestras, tanto por comparación visual como asistida por la aplicación COFECHA (Holmes, 1983). Este programa verifica la sincronización entre las diferentes series individuales y la serie maestra, aplicando estadística básica para

hallar correlaciones entre segmentos de series estandarizadas, advirtiendo de posibles anomalías o problemas, como anillos ausentes, o valores fuera de rango. COFECHA sirve además para controlar la calidad de los datos, tanto de medición del ancho de los anillos como la datación cruzada.

\section{RESULTADOS}

Siguiendo los procedimientos descritos en la sección anterior, de las muestras de los algarrobos modernos se obtuvo una serie dendrocronológica, a la que se puede asignar un año calendárico, puesto que las muestras poseen corteza, y el anillo bajo esta corresponde al año de corte, registrado durante la recolección de las muestras.

De las muestras arqueológicas se obtuvo una serie dendroarqueológica que aún no pueden fecharse, constituyendo una serie flotante (Douglass, 1941). Sin embargo, constituye una cronología relativa, y puede dar información sobre la variación climática anual.

\section{1. Serie dendrocronológica moderna}

La serie dendrocronológica de 95 años de árboles modernos de Casma comprende una serie de valores de anchos de anillos obtenida de las secciones de algarrobos con fecha de corte del año 2002. Esta serie se contrastó con datos de calibración compuestos por parámetros climáticos disponibles para la costa norte, como la lluvia y la temperatura del agua del mar (TSM), tomadas de la base de datos de parámetros climáticos de la Facultad de Ingeniería de la UDEP. 
La figura 9 muestra que las variaciones medidas en el ancho de los anillos de los algarrobos corresponden estrechamente a las variaciones anuales en precipitación y TSM. Esto confirma, en primer lugar, el carácter anual de los anillos del algarrobo. Asimismo, la influencia de los factores climáticos en el crecimiento del algarrobo se aprecia claramente en las variaciones proporcionales en el ancho de los anillos, lo que comprueba que esta especie es muy sensible a estos factores limitantes. Adicionalmente, el cuadro 1 muestra los índices de correlación ( $r$ de Pearson) entre la serie de ancho de anillos y varios parámetros climáticos disponibles. Se aprecia que la serie tiene correlaciones significativas con estos parámetros climáticos, inclusive de regiones alejadas, como Piura. Es decir, se satisfacen todas las condiciones básicas de la dendrocronología (Stokes \& Smiley, 1968; Rodríguez \& Fernández, 2009; Speer, 2010).

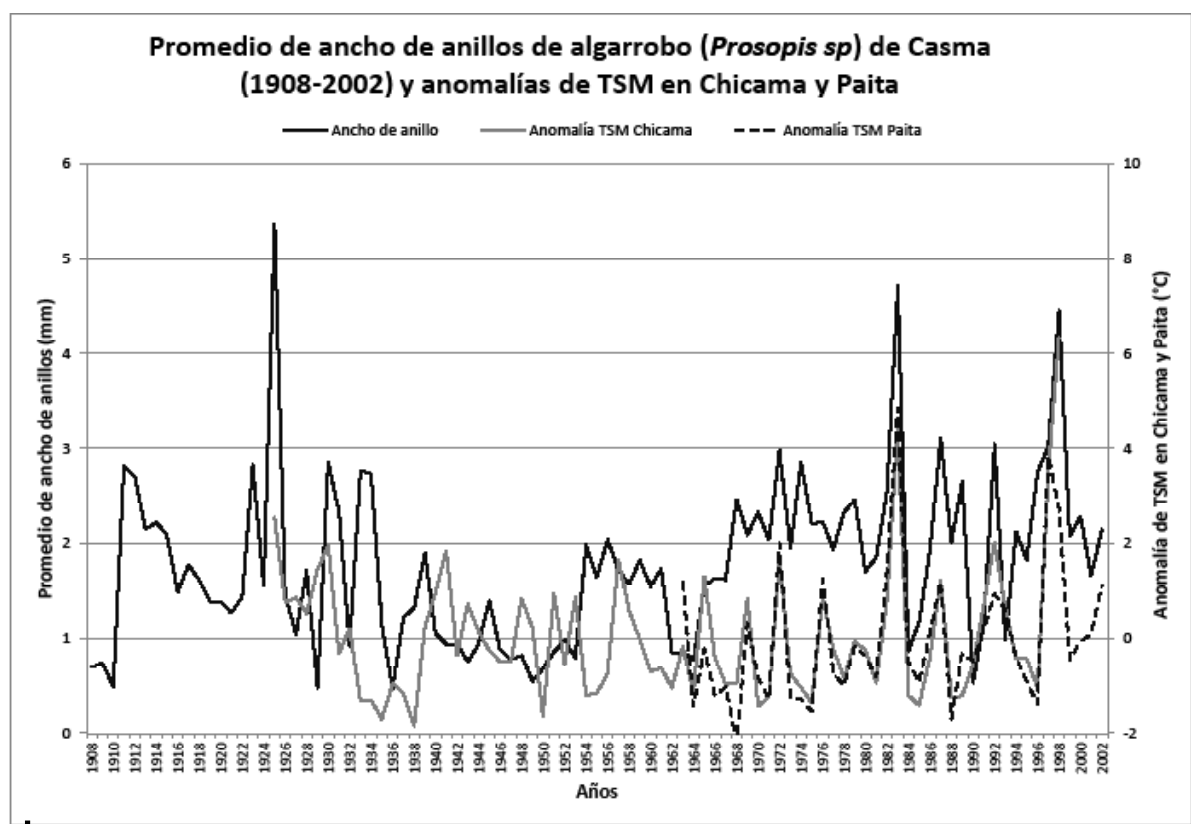

Figura 9 - Comparación gráfica entre la serie dendrocronológica moderna de Casma y series climáticas (anomalías de TSM en Chicama y Paita)

(C) Rodolfo Rodríguez

Los parámetros empleados para la calibración de estos datos, como la precipitación y la temperatura del agua del mar (TSM), son muy sensibles a la ocurrencia del fenómeno El Niño. En esta serie dendrocronológica se registra muy claramente la señal climática de eventos de El Niño de gran magnitud de los años 1925, 1983 y 1998, que dieron lugar a la generación de anillos de crecimiento significativamente más anchos: estos superan los 4,0 mm de espesor, mientras que el promedio de ancho de los anillos de toda la serie es 1,8 mm, y en el resto de anillos los otros picos no sobrepasan los $3 \mathrm{~mm}$. 
Cuadro 1 - Índices de correlación* entre la serie dendrocronológica moderna y series climáticas

\begin{tabular}{|c|c|c|c|}
\hline \multirow{4}{*}{ Serie moderna } & Series climáticas & Índice & $\begin{array}{c}\text { Rango de } \\
\text { años }\end{array}$ \\
& Lluvia anual cn Casma & 0.5 & $1966-2002$ \\
\cline { 2 - 4 } & Anomalía de TSM en Chicama & 0.46 & $1925-1998$ \\
\cline { 2 - 4 } $\begin{array}{c}\text { Promedio de } \\
\text { ancho de } \\
\text { anillos }\end{array}$ & Lluvia anual en Piura & 0.69 & $1957-2002$ \\
\cline { 2 - 4 } & Anomalía de TSM Paita & 0.55 & $1963-2002$ \\
\cline { 2 - 4 } & Anomalía TSM NINO3 (Promedio DEF) & 0.4 & $1950-2002$ \\
\cline { 2 - 4 } & Serie Palo Santo (Bursera (iraveolens) & 0.68 & $194 /-2001$ \\
\hline
\end{tabular}

* r de Pearson

La serie dendrocronológica obtenida de algarrobos modernos del valle de Casma tiene una correlación alta $(0,68, r$ de Pearson) con una serie dendrocronológica obtenida de palo santo (Bursera graveolens) del bosque seco de la región Piura (Rodríguez \& Fernández, 2009). Esto indica una señal climática fuertemente compartida entre ambos registros, pese a la distancia entre estas regiones. La comparación corresponde a series comunes de anchos de anillos de ambas especies de 56 valores para cada una del lapso entre 1946 y 2001, como se muestra en la figura 10.

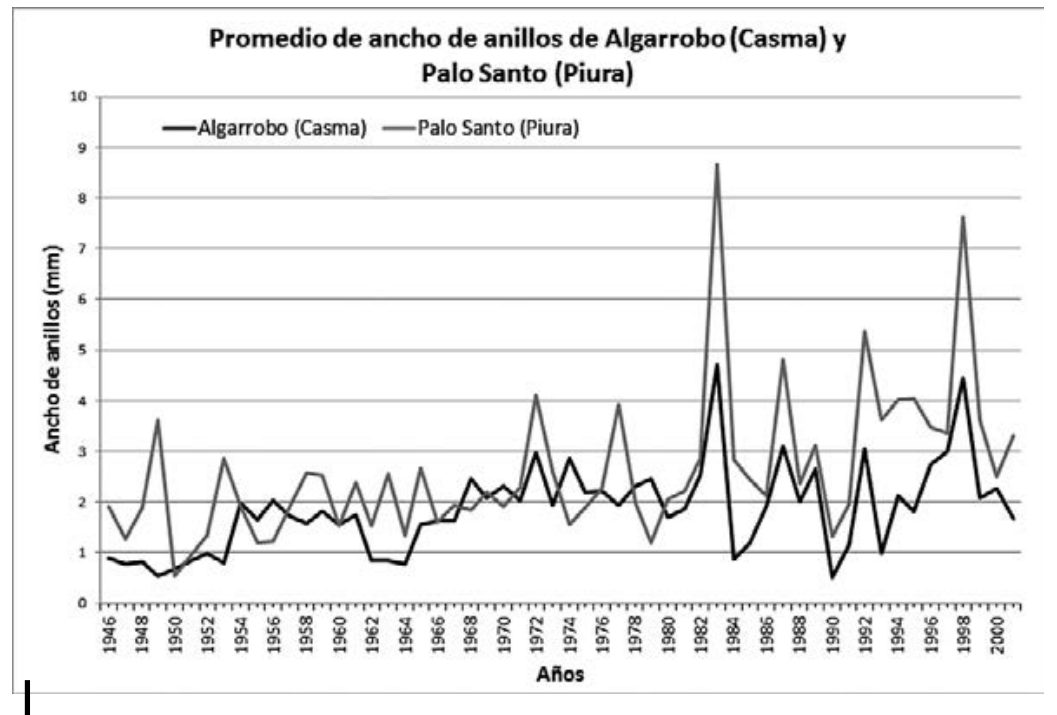

Figura 10 - Comparación gráfica de series de anchos de anillos de Algarrobo de Casma y de Palo Santo de Piura para el lapso 1946-2001 () Rodolfo Rodríguez) 


\section{2. Serie dendroarqueológica de Chankillo}

En cuanto a la serie prehispánica de Chankillo, tiene una extensión de 63 años (fig.11). La correlación ( $r$ de Pearson) promedio entre las muestras es un valor alto $(0,53)$. Al no existir una secuencia maestra contra la cual compararla, no se puede asignar años calendáricos a esta secuencia, por lo que se trata de una serie flotante. Sin embargo, según las fechas ${ }^{14} \mathrm{C}$ obtenidas del anillo externo, o de corte, la serie se ubica aproximadamente entre los años 375 y 120 CalBC, lo cual es consistente con el resto de fechas radiocarbónicas de Chankillo (Ghezzi, 2006). Se observan varios anillos con un ancho promedio notablemente mayor al resto (más de 5,0 $\mathrm{mm}$ de espesor), mientras que el promedio de ancho de anillos de la serie es 2,8 mm, y en el resto de anillos los picos no sobrepasan los 4,0 mm. El ancho del anillo para estos años podría estar relacionado con eventos de El Niño muy fuertes. Asimismo, el ancho promedio de los anillos de esta serie prehispánica es $55 \%$ mayor al de la serie moderna.

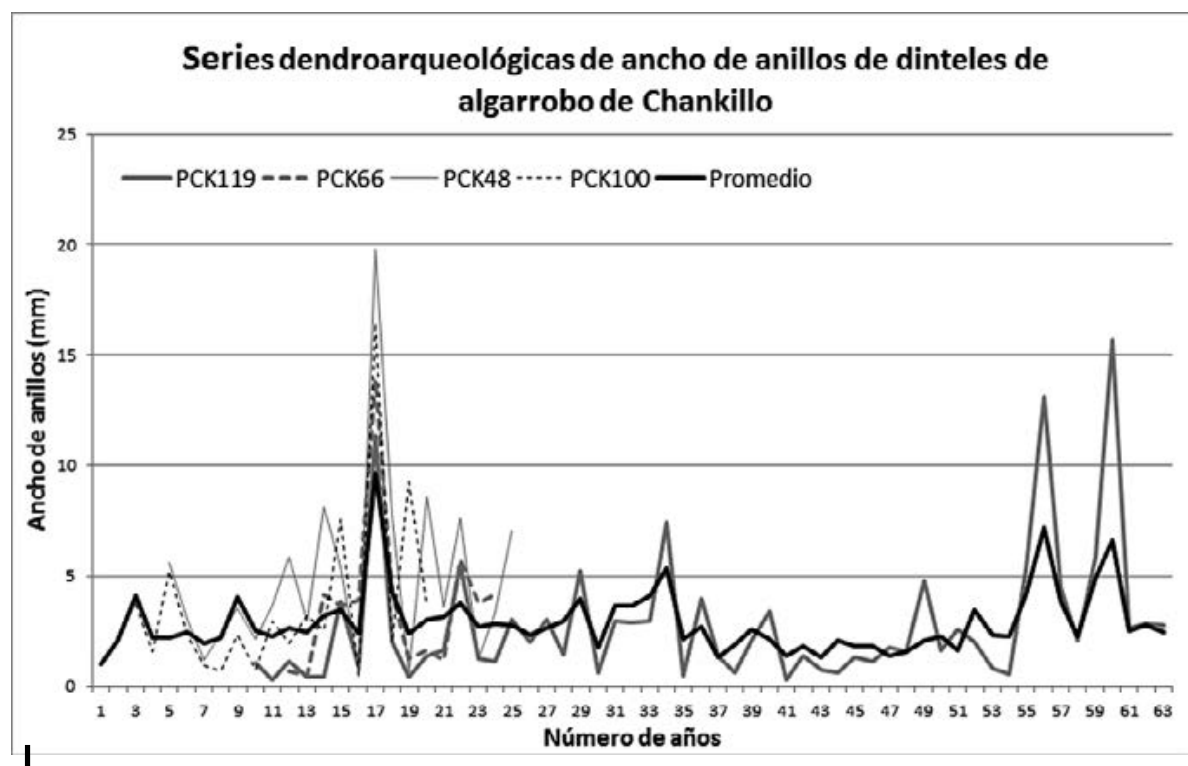

Figura 11 - Gráfico de series dendroarqueológicas de ancho de anillos de madera de algarrobo procedentes de los dinteles prehispánicos de Chankillo

\section{DISCUSIÓN}

En primer lugar, se ha probado la presencia de anillos visibles, anuales y limitados por parámetros climáticos de alcance regional en los algarrobos modernos muestreados en el bosque seco aledaño a Chankillo. Estas son condiciones básicas de la dendrocronología. 
Sorprendentemente, la serie moderna guarda la correlación más alta con parámetros climáticos de la región Piura. Esto se explica porque el sur del Ecuador y el extremo norte del Perú son la zona continental de Sudamérica más impactada por el fenómeno climático El Niño. Punta Balcones (Piura) es, por ejemplo, la zona más occidental del continente sobre el Océano Pacifico, a lo que se debe la alta sensibilidad de la región a este fenómeno climático. Al registrar más fuertemente la señal climática de El Niño, las correlaciones entre los datos de Piura y otras regiones serán naturalmente más altas.

La alta correlación entre territorios tan distantes indica que el clima que determina el grosor de los anillos del algarrobo tiene un alcance macrorregional. Esto tiene implicancias importantes cuando se aplica el principio de uniformitarianismo y se usa el resultado de la serie moderna para validar la aplicación de la dendrocronología a la época prehispánica. En primer lugar, se puede asumir que los algarrobos en la época de ocupación de Chankillo tenían el mismo comportamiento, es decir, sus anillos de crecimiento visibles eran también anuales y limitados por parámetros climáticos de alcance regional. En segundo lugar, se puede asumir además que algarrobos de época prehispánica en una región amplia, de Piura a Áncash y quizás más al sur, que básicamente comparten el mismo clima, respondían a este ambiente de modo similar a los algarrobos modernos, y su crecimiento estaba altamente correlacionado. Esto permitirá elaborar a futuro una serie dendrocronológica maestra para la costa, con datos como los que estamos recogiendo en Piura, Lambayeque, La Libertad y Ancash, con el objetivo de elaborar a corto plazo secuencias flotantes a fecharse mediante wiggle-matching (Kojo et al., 1994; Ghezzi et al., 2015).

Como se ha advertido, el ancho promedio de los anillos de la serie prehispánica es mayor al de la serie de árboles modernos. La interpretación más directa es que esto indica un clima en general más favorable (mayor disponibilidad de agua) para el crecimiento del algarrobo durante la época de ocupación de Chankillo. Sin embargo, no puede descartarse otras posibilidades. Una de ellas es que los árboles con los que se fabricaron los dinteles hayan sido gestionados mediante el riego, la fertilización, la poda y otros cuidados, para maximizar la obtención de madera. A ello podría deberse un crecimiento más acelerado que el que se ve hoy en día, sin intervención del hombre, en este mismo bosque seco. Otra opción es que la presencia de un fenómeno de El Niño muy fuerte hacia el inicio de la serie (el ancho promedio de anillos del año 17 casi duplica los otros picos en esta serie, y en la moderna) haya tenido efectos duraderos en el crecimiento posterior de los árboles (como parece suceder entre los años 18 y 34 en la serie), elevando de ese modo el ancho promedio de los anillos de toda la serie.

Pese a ser una secuencia flotante, esta permite establecer una cronología relativa en el Templo Fortificado de Chankillo. Al organizar las muestras de acuerdo a la portada de procedencia (fig. 12), y tomar en cuenta el anillo externo como indicador de la fecha de corte del árbol, se observa que la mayoría de los dinteles en cada portada tiene fechas de corte muy cercanas entre sí, dentro de los primeros cinco años de la serie cronológica. Las fechas de corte son un indicador 


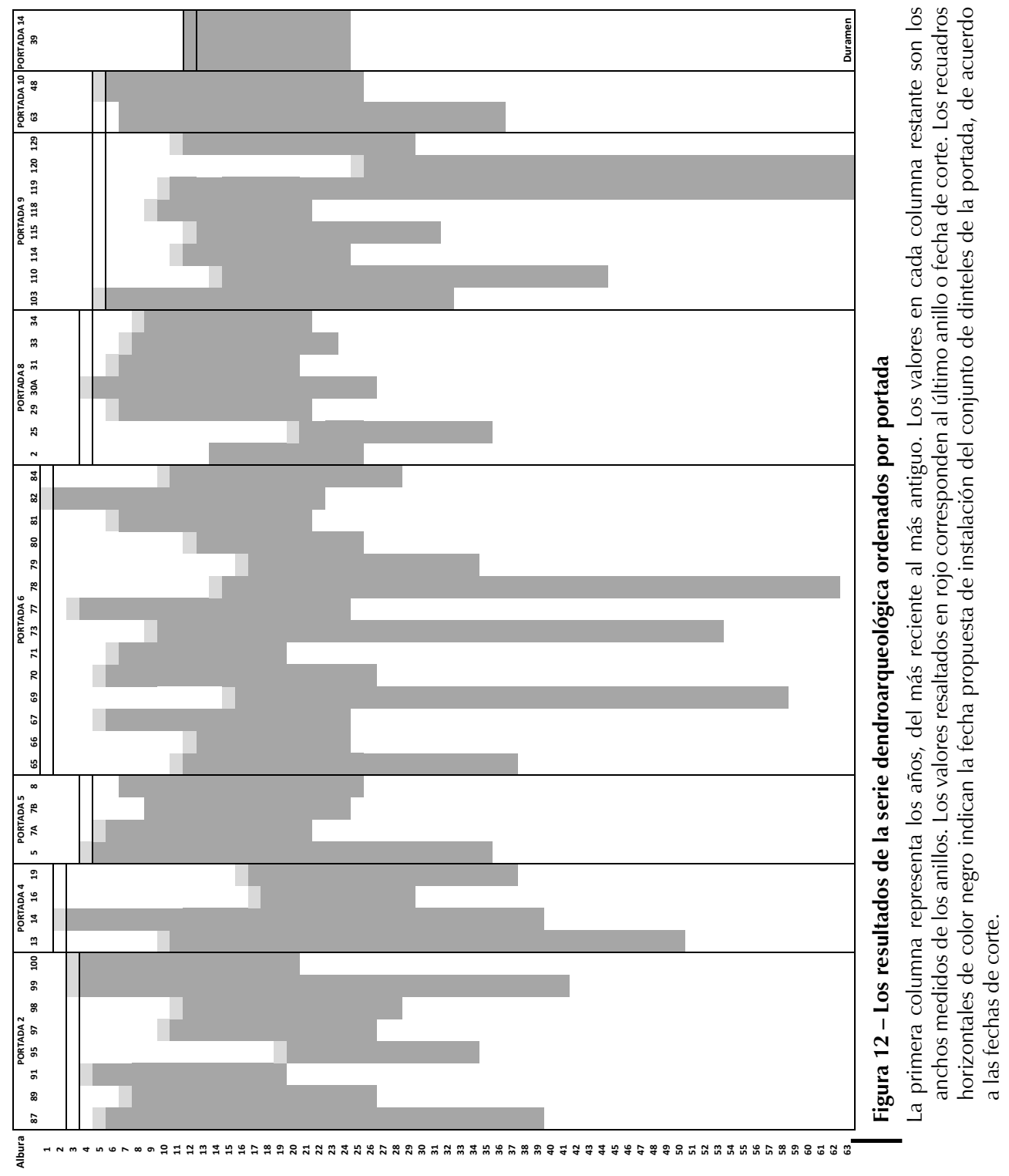


bastante razonable de la fecha aproximada de instalación del dintel, y por lo tanto la gran mayoría de dinteles en el Templo Fortificado se habrían fabricado e instalado sobre los muros en un lapso corto, de solo cinco años.

No se puede concluir necesariamente que la instalación de los dinteles coincide con la fecha de construcción de las portadas, y menos de las murallas en las que se encuentran. Sin embargo, la inusual sincronía en las fechas de instalación de todos estos dinteles, pese a que proceden de diferentes partes del edificio, sugiere razonablemente que se trata de un solo gran evento constructivo y que todo el Templo Fortificado fue erigido en un lapso relativamente corto.

Es notable que se haya creado la primera secuencia dendroarqueológica de los Andes Centrales. En el futuro es necesario construir, con muestras de otras regiones y periodos, de los primeros habitantes y construcciones al presente, una secuencia dendrocronológica maestra para la costa peruana. La secuencia maestra permitirá obtener fechados calendáricos, reconstrucciones climáticas y otras aplicaciones típicas de la dendrocronología a partir del algarrobo, que es un árbol común en la costa peruana, y cuya madera es un hallazgo frecuente en contextos arqueológicos.

\section{Agradecimientos}

El Instituto Francés de Estudios Andinos, a través de su programa de Becas Andinas, hizo posible esta fase de la investigación. National Science Foundation, Laboratory of Tree-ring Research, World Monuments Fund, Cooperative Institute for Research in Environmental Sciences (CIRES), y Tree-ring Lab of LDEO (EEUU), Inter-American Institute for Global Change (Brasil) e Instituto Geofísico del Perú (IGP), brindaron fondos y asistencia técnica en diversas etapas de la investigación dendrocronológica de los autores. Finalmente, el Fondo de Investigación en Ciencia y Tecnología (FINCyT), a través de su programa Proyectos de Investigación Básica 2014, financia el desarrollo de la dendrocronología en la costa norte con fines de reconstrucción paleoclimática.

\section{Referencias citadas}

BAILLIE, M. G. L., 1982 - Tree-ring dating and archaeology, 274 pp.; Chicago: University of Chicago Press.

BAILLIE, M. G. L., 1995 - A slice through time: Dendrochronology and precision dating, 176 pp.; Bath: Psychology Press.

BANNISTER, B., 1969 - Dendrochronology. In: Science in archaeology. A survey of progress and research (D. R. Brothwell \& E. S. Higgs, eds.): 191-205; London: Thames and Hudson.

BILLAMBOZ, A., 1992 - Tree-ring analysis in archaeodendrological perspective: The structural timber from the south west German lake dwellings. In: Tree-rings and environment (T. S. Bartholin, ed.): 34-40; Lund University, Sweden: Department of Quaternary Geology. Proceedings of the international symposium, Ystad, Sweden, 3-9 september, 1990. 
BILLAMBOZ, A., 2003 - Tree-rings and wetland occupation in southwest Germany between 2000 and 500 bc: Dendroarchaeology beyond dating in tribute to $\mathrm{FH}$ Schweingruber. Tree-ring Research, 59 (1): 37-49.

BONINSEGNA, J. A., ARGOLLO, J., ARAVENA, J., BARICHIVICH, J., CHRISTIE, D., FERRERO, M., LARA, A., LE QUESNE, C., LUCKMAN, B. \& MASIOKAS, M., 2009 Dendroclimatological reconstructions in South America: A review. Palaeogeography, Palaeoclimatology, Palaeoecology, 281 (3): 210-228.

BRIENEN, R. J. W., HELLE, G., PONS, T. L., GUYOT, J. L. \& GLOOR, M., 2012 - Oxygen isotopes in tree rings are a good proxy for Amazon precipitation and El NiñoSouthern Oscillation variability. Proceedings of the National Academy of Sciences, 109 (42): 16957-16962.

BURGER, R. L., 1995 - Chavín and the origins of Andean civilization, 248 pp.; London: Thames and Hudson.

CHRISTIE, D. A., LARA, A., BARICHIVICH, J., VILLALBA, R., MORALES, M. S. \& CUQ, E., 2009 - El Niño-Southern Oscillation signal in the world's highest-elevation tree-ring chronologies from the Altiplano, Central Andes. Palaeogeography, Palaeoclimatology, Palaeoecology, 281 (3): 309-319.

COOK, E. R. \& HOLMES, R. L., 1984 - Program ARSTAN users manual, 15 pp.; Tucson: Laboratory of Tree-Ring Research, University of Arizona.

ČUFAR, K., 2007 - Dendrochronology and past human activity: A review of advances since 2000. Tree-ring Research, 63 (1): 47-60.

DEAN, J. S., 1978 - Tree-ring dating in archaeology. University of Utah Miscellaneous Paper, 19 (24): 129-163.

DEAN, J. S., 1997 - Dendrochronology. In: Chronometric dating in archaeology (R. E. Taylor \& M. J. Aitken, eds.): 31-64; Nueva York: Plenum Press.

DEAN, J. S., MANNING, S. W. \& BRUCE, M. J., 2009 - One hundred years of dendroarchaeology: Dating, human behavior, and past climate. In: Tree-rings, kings, and old world archaeology and environment: Papers presented in honor of Peter lan Kuniholm (S. W. Manning \& M. J. Bruce, eds.): 25-32; Oxford, Oakville: Oxford Book.

DOUGLASS, A. E., 1919 - Climatic cycles and tree-growth: A study of the annual rings of trees in relation to climate and solar activity; Washington: Carnegie Institution of Washington.

DOUGLASS, A. E., 1929 - The secret of the Southwest solved by talkative tree rings. National Geographic Magazine, 56: 736-770.

DOUGLASS, A. E., 1937 - Tree rings and chronology, 36 pp.; Tucson, Arizona: University of Arizona.

DOUGLASS, A. E., 1941 - Crossdating in dendrochronology. Journal of Forestry, 39 (10): 825-831.

FERGUSON, C. W., 1970 - Concepts and techniques of dendrochronology. In: Scientific methods in medieval archaeology (R. Berger, ed.): 183-200; Los Angeles: University of California Press.

FRITTS, H. C., 1976 - Tree rings and climate, 567 pp.; London: Academic Press.

GHEZZI, I., 2006 - Religious warfare at Chankillo. In: Andean archaeology III (W. Isbell \& H. Silverman, eds.): 67-84; Nueva York: Springer.

GHEZZI, I., 2007 - La naturaleza de la guerra prehispánica temprana: La perspectiva desde Chankillo. Revista Andina, 44: 199-225. 
GHEZZI, I. \& GUADALUPE GÓMEZ, E., 2013 - Evidencias geológicas en el complejo astronómico Chankillo (Casma, Ancash). Revista del Instituto de Investigación de la Facultad de Ingeniería Geológica, Minera, Metalurgica y Geográfica, 16 (31): 1-14.

GHEZZI, I. \& RUGGLES, C., 2007 - Chankillo: A 2300-year-old solar observatory in coastal Peru. Science, 315 (5816): 1239-1243.

GHEZZI, I., TOWNER, R. H., WINTERS, R. \& RODRÍGUEZ, R., 2002 - Dendroarchaeology at Chankillo. Ponencia presentada en Society for American Archaeology 67th. Annual Meeting; Denver, Colorado.

GHEZZI, I., MABRES, A., RODRÍGUEZ, R., CRUZ, G. \& SALAZAR, P. C., 2015 - Estudio de parámetros biológicos en Prosopis sp. como un registro paleoclimático: Fundamentos y uso de nuevos métodos de medición dendrocronológica. Proyecto de Investigación Básica FINCYT/CONCYTEC, Perú.

GRISSINO-MAYER, H. D., 2009-An introduction to dendroarchaeology in the southeastern United States. Tree-ring Research, 65 (1): 5-10.

HOGG, A. G., HUA, Q., BLACKWELL, P. G., NIU, M., BUCK, C. E., GUILDERSON, T. P., HEATON, T. J., PALMER, J. G., REIMER, P. J. \& REIMER, R. W., 2013 - Shcal13 southern hemisphere calibration, 0-50,000 years cal bp. Radiocarbon, 55 (2): 1-15.

HOLMES, R. L., 1983 - Computer-assisted quality control in tree-ring dating and measurement. Tree-Ring Bulletin, 43: 69-78.

KOJO, Y., KALIN, R. M. \& LONG, A., 1994 - High-precision "wiggle-matching" in radiocarbon dating. Journal of Archaeological Science, 21 (4): 475-479.

LÓPEZ, B., SABATÉ, S., GRACIA, C. \& RODRÍGUEZ, R., 2005 - Wood anatomy, description of annual rings, and responses to ENSO events of Prosopis pallida h.B.K., a widespread woody plant of arid and semi-arid lands of Latin America. Journal of Arid Environments, 61 (4): 541-554.

MCCARROLL, D. \& LOADER, N. J., 2004 - Stable isotopes in tree rings. Quaternary Science Reviews, 23 (7): 771-801.

MORALES, M. S., NIELSEN, A. E. \& VILLALBA, R., 2013 - First dendroarchaeological dates of prehistoric contexts in South America: Chullpas in the central andes. Journal of Archaeological Science, 40 (5): 2393-2401.

MORALES, M. S., VILLALBA, R., GRAU, H. R., VILLAGRA, P. E., BONINSEGNA, J. A., RIPALTA, A. \& PAOLINI, L., 2001 - Potencialidad de Prosopis ferox griseb (leguminosae, subfamilia: Mimosoideae) para estudios dendrocronológicos en desiertos subtropicales de alta montaña. Revista Chilena de Historia Natural, 74 (4): 865-872.

MORALES, M., CHRISTIE, D., VILLALBA, R., ARGOLLO, J., PACAJES, J., SILVA, J., ÁLVAREZ, C., LLANCABURE, J. \& SOLIZ GAMBOA, C., 2012 - Precipitation changes in the South american altiplano since 1300 ad reconstructed by tree-rings. Climate of the Past, 8 (2): 653-666.

PALACIOS, R. A., BURGHARDT, A. D., FRÍAS-HERNÁNDEZ, J. T., OLALDE-PORTUGAL, V., GRADOS, N., ALBAN, L. \& MARTíNEZ-DE LA VEGA, O., 2012 - Comparative study (aflp and morphology) of three species of Prosopis of the section algarobia: P. Juliflora, p. Pallida, and p. Limensis. Evidence for resolution of the "p. Pallida-p. Juliflora complex". Plant systematics and evolution, 298 (1): 165-171.

PASIECZNIK, N. M., FELKER, P., HARRIS, P. J. C., HARSH, L. N., CRUZ, G., TEWARI, J. C., CADORET, K. \& MALDONADO, L. J., 2001 - The Prosopis julifora-Prosopis pallida complex: A monograph, 172 pp.; Coventry, UK: Henry Doubleday Research Association. 
RAMSEY, C. B., 1995 - Radiocarbon calibration and analysis of stratigraphy: The oxcal program. Radiocarbon, 37 (2): 425-430.

RAMSEY, C. B., 2001 - Development of the radiocarbon program oxcal. Radiocarbon, 43 (2A): 355-363.

REIMER, P. J., BARD, E., BAYLISS, A., BECK, J. W., BLACKWELL, P. G., BRONK RAMSEY, C., BUCK, C. E., CHENG, H., EDWARDS, R. L. \& FRIEDRICH, M., 2013 - Intcal13 and Marine13 radiocarbon age calibration curves 0-50,000 years cal bp. Radiocarbon, 55 (4): 1869-1887.

RIVERA, M. A., R., J. M. \& SHEA, D. E., 2010 - Dendrocronología en la Pampa del Tamarugal, desierto de Atacama, norte de Chile. Diálogo Andino, 36: 33-50.

RODRÍGUEZ, R. \& FERNÁNDEZ, R., 2009 - Dendrocronología básica, 67 pp.; Piura: Gobierno Regional de Piura.

RODRÍGUEZ, R., WOODMAN, R., BALSLEY, B., MABRES, A. \& PHIPPS, R., 1993 Avances sobre estudios dendrocronológicos en la región costera norte del Perú para obtener un registro pasado del fenómeno El Niño. Bulletin de I'Institut Français d'Études Andines, 22: 267-281.

RODRÍGUEZ, R., MABRES, A., LUCKMAN, B., EVANS, M., MASIOKAS, M. \& EKTVEDT, T. M., 2005 - "El Niño" events recorded in dry-forest species of the lowlands of northwest Peru. Dendrochronologia, 22 (3): 181-186.

SALAZAR, P. C., 2013 - Estrategia de manejo para el bosque seco de algarrobo en el área protegida de Chankillo. Informe presentado al Proyecto Chankillo; Casma.

SALCEDO, L. E., 2011 - Tempus solaris: Algunas nociones sobre la actividad solar, las variaciones seculares del carbono-14 atmosférico y los estadios del isótopo de carbono, 190 pp.; Lima: Intersur.

SASS-KLAASSEN, U., 2002 - Dendroarchaeology: Successes in the past and challenges for the future. Dendrochronologia, 20 (1): 87-93.

SCHIFFER, M. B., 1986 - Radiocarbon dating and the "old wood" problem: The case of the Hohokam chronology. Journal of Archaeological Science, 13 (1): 13-30.

SCHWEINGRUBER, F. H., 1988 - Tree rings: Basics and applications of dendrochronology, 276 pp.; Dordrecht, Boston: D. Reidel Publishing Company.

SPEER, J. H., 2010 - Fundamentals of tree-ring research, 333 pp.; Tucson: University of Arizona Press.

STOKES, M. A. \& SMILEY, T. L., 1968 - An introduction to tree-ring dating, 73 pp.; Chicago: University of Chicago Press.

TOWNER, R. H., 2001 - Dendroarchaeology. In: Encyclopedia of Quaternary Science, IKS (Elias \& C. Mock, eds.): 2307-2315; Amsterdam: Elsevier.

TOWNER, R. H., 2002 - Archeological dendrochronology in the Southwestern United States. Evolutionary Anthropology: Issues, News, and Reviews, 11 (2): 68-84.

VILLALBA, R., VILLAGRA, P. E., BONINSEGNA, J. A., MORALES, M. S. \& MOYANO, V., 2000 - Dendroecología y dendroclimatología con especies del género Prosopis en Argentina. Multequina, 9 (2): 1-18. 